\title{
Les intoxications aiguës à la chloroquine : aspects cliniques et analytiques
}

\section{Acute chloroquine poisoning : clinical and analytical aspects}

\section{Aurélie SERVONNET*, Hervé DELACOUR, Hélène THEFENNE, Véronique GARDET}

Fédération de Biologie Clinique, Hôpital d'Instruction des Armées Robert PICQUE, 331, Route de Toulouse - 33140 VILLENAVE D'ORNON

*Auteur à qui adresser la correspondance : Aurélie SERVONNET, Fédération de Biologie Clinique, Hôpital d'Instruction des Armées Robert PICQUE, 331, Route de Toulouse - 33140 VILLENAVE D'ORNON Tél : 0556847302 - Fax : 0556847495 - E-mail : a_servonnet@yahoo.fr

(Reçu le 23 décembre 2004 ; accepté après modifications le 16 mars 2005)

\section{$R \hat{E} S U M E ́$}

Molécule à index thérapeutique étroit, la chloroquine est l'antimalarique le plus utilisé dans le monde. Elle est à l'origine d'intoxications aiguës graves rencontrées dans les zones impaludées et plus rarement en Europe. Leur sévérité est. essentiellenent liée aux troubles cardiovasculaires provoqués par son action quinidine-like. Le traitement thérapeutique fait appel principalement au diazépam, à l'adrénaline et à la ventilation artificielle. Le protocole de traitement spécifique ne doit être appliqué dans son intégralité qu'en cas d'intoxications graves et non systématiquement. Son efficacité sera augmentée en cas de prise en charge pré-hospitalière précoce. La gravité des intoxications est évaluée à l'aide de différents paramètres cliniques (pression artérielle systolique, troubles du rythme), biologiques (hypokaliémie) et toxicologiques (chloroquinémie). Les laboratoires hospitaliers possèdent donc un rôle fondamental en affirmant le diagnostic et en évaluant la gravité de l'intoxication par le dosage de la chloroquine, réalisé préférentiellement sur sang total. Les techniques analytiques utilisées sont essentiellement spectroscopiques (UV) ou chromatographiques (C.L.H.P. ou C.P.G.), Malheureusenent, ces dernières, méthodes de référence pour les analyses médico-légales, sont difficilement adaptées à l'urgence du fait de leur délai de rendu de résultat.

\section{MOTS-CLÉS}

Chloroquine, intoxication, toxicologie analytique, protocole thérapeutique.

\section{SUMMARY}

Chloroquine is the mainly and most frequently drug used as antimalaric in the world, in spite of the extension of resistance phenomena. Due to the narrow margin between therapeutic and toxic dose, fatal chloroquine poisoning come to notice from time to time in Africa and more exceptionally in. Europe. Acute intoxication is responsible of grave cardiovascular disturbances, which may be rapidly life threatening. Cardiovascular toxicity is linked to a potent membrane-stabilising effect provoked by its quinidine-like action. Treatment is based on diazepam, adrenalin, and assisted ventilation. Its effectiveness will be increased in case of premature coverage. Seriousness of poisoning is estimated by means of various clinical (systolic blood pressure, rhythm. disorders), biological (hypokalaemia) and toxicological (chloroquinaemia) parameters. Thus, hospital laboratories possess a fundamental role by asserting diagnosis and by estimating poisoning's gravity by chloroquine's determination preferentially in whole blood. Analytical techniques are essentially spectrophometric (UV) or chromatographic (HPLC or GPC). Unfortunately, chromatographic methods are adapted with difficulty to the urgency because of their delay of result's depiction.

\section{KEY-WORDS}

Chloroquine, poisoning, analytical toxicology, therapeutic protocol. 


\section{Introduction}

La chloroquine (7-chloro-4-[[4-(diethylamino)-1méthylbutyl]amino]quinoléine) est une amino-4-quinoléine de synthèse découverte en 1934 par des chercheurs allemands. Indiquée dans la chimioprophylaxie et dans le traitement curatif du paludisme, elle est également prescrite en rhumatologie et en dermatologie pour traiter la polyarthrite rhumatoïde et certains lupus (1-2). En France, elle est retrouvée au sein de plusieurs spécialités pharmaceutiques seule ou associée au proguanil (tableau I). Ses effets indésirables sont rares dans le cadre d'une chimioprophylaxie (risque estimé inférieur à $1 / 100$ 000) et apparaissent principalement en cas de traitements prolongés (3). A l'inverse, les intoxications aiguës, par surdosage ou lors de tentative d'autolyse, sont marquées par la survenue d'une symptomatologie grave potentiellement fatale. Si elles sont relativement fréquentes dans la zone d'endémie palustre (4-5), elles sont devenues rares voire exceptionnelles en Europe (6-11). Cependant, au milieu des années 80 , une augmentation de l'incidence des intoxications aiguës a été observée en métropole. Elle faisait suite à la publication d'un ouvrage, heureusement rapidement interdit à la vente, mentionnant la chloroquine comme médicament à utiliser en cas de tentative de suicide (12). La parution d'un livre similaire en Allemagne fut accompagnée également de passages à l'acte (13). De plus, la chloroquine est très fréquemment retrouvée dans les «armoires à pharmacie» des familles ayant séjournées dans une zone impaludée. Dans cette revue, après quelques rappels sur la pharmacocinétique, nous développerons les aspects cliniques et analytiques des intoxications aiguës à la chloroquine.

\section{Rappels sur la pharmacoci- nétique}

Après une prise orale à dose thérapeutique, la chloroquine est absorbée au niveau du grêle proximal de façon rapide et pratiquement complète (biodisponibilité évaluée entre $78 \%$ et $89 \%$ selon les études). Le pic de concentration plasmatique est atteint en moyenne entre $1 \mathrm{H} 30$ et $3 \mathrm{H} 00$ après la prise (3). Lors d'une intoxication, l'ingestion massive diminue l'évacuation gastrique et la mobilité digestive et augmente les sécrétions acides, ce qui est à l'origine d'un ralentissement de l'absorption. Il n'est pas rare, dès lors, d'observer une augmentation de la concentration plasmatique jusqu'à 10 hểres après l'ingestion. De même, des quantités importantes de chloroquine peuvent être retrouvées dans le liquide gastrique jusqu'à plusieurs jours après l'intoxication en l'absence de traitement évacuateur (14).

La chloroquine possède un volume de distribution très élevé, autour de $200 \mathrm{~L} . \mathrm{kg}^{-1}$ en tenant compte de la concentration dans le sang total et entre 200 et 800 L. $\mathrm{kg}^{-1}$ en se référant aux concentrations plasmatiques. Ceci s'explique par sa forte fixation protéique (environ $60 \%$ ) et tissulaire (cœur, moelle osseuse, rate, leucocytes). Lysosomotrope, elle pénètre dans les érythrocytes pour atteindre des concentrations 3 à 6 fois supérieures à celles retrouvées dans le plasma (3).

Le métabolisme hépatique par déalkylation conduit principalement à trois métabolites pharmacologiquement actifs: la déséthylchloroquine, la bi-déséthylchloroquine et la 7-chloro-4-aminoquinoléine (figure 1). $70 \%$ de la chloroquine est éliminée sous forme inchangée au niveau urinaire avec une demi-vie d'élimination située entre 20 et 60 jours. L'élimination urinaire représente $10 \%$ de la dose ingérée en 48 heures et $30 \%$ en 7 jours.

Tableau I : Liste des spécialités pharmaceutiques contenant de la chloroquine commercialisées en France.

\begin{tabular}{|c|c|c|}
\hline & Nom des spécialités & Forme galénique é présentation \\
\hline $\begin{array}{l}\text { Chloroquine } \\
\text { (sulfate) }\end{array}$ & $\begin{array}{l}\text { NIVAQUINE }^{\otimes} \\
\text { (RHONE POULENC ROHRER) }\end{array}$ & $\begin{array}{l}\text { Comprimés à } 100 \mathrm{mg} \text {, boîte de } 20 \text { ou } 100 \\
\text { Comprimés à } 300 \mathrm{mg} \text {, boîte de } 4 \\
\text { Sirop à } 5 \mathrm{mg} \cdot \mathrm{mL}^{-1} \text {, flacon de } 150 \mathrm{~mL} \\
\text { Solution injectable à } 50 \mathrm{mg} \cdot \mathrm{mL}^{-1} \text {, boîte de } 5 \text { ampoules de } 2 \mathrm{~mL}\end{array}$ \\
\hline $\begin{array}{l}\text { Chloroquine } \\
\text { (phosphate) } \\
+ \text { Proguanil }(200 \mathrm{mg})\end{array}$ & $\begin{array}{l}\text { SAVARINE }^{\circ} \\
\text { (ZENECA PHARMA) } \\
\text { NOPALU }^{\odot} \\
\text { (SERVICE DE SANTÉ DES ARMÉES) }\end{array}$ & $\begin{array}{l}\text { Comprimés à } 100 \mathrm{mg} \text {, boîte de } 28 \\
\text { Gélules à } 136 \mathrm{mg} \text {, boîte de } 20 \text { ou } 100\end{array}$ \\
\hline
\end{tabular}




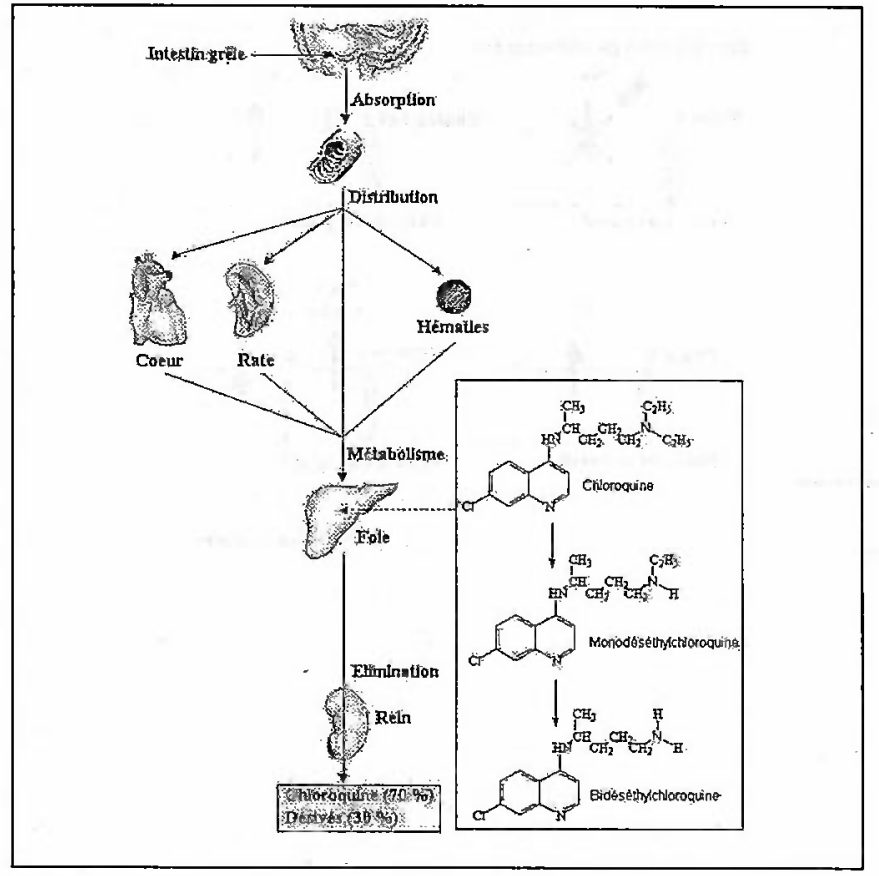

Figure 1 : Représentation schématique de la pharmacocinétique de la chloroquine.

La chloroquine possède un index thérapeutique étroit. En prophylaxie antipalustre, la dose thérapeutique recherchée est de $10 \mathrm{mg} \cdot \mathrm{kg}^{-1}$, soit une concentration plasmatique de l'ordre de 0,01 à $0,4 \mathrm{mg} \cdot \mathrm{L}^{-1}$. La dose toxique est évaluée à $20 \mathrm{mg} \cdot \mathrm{kg}^{-1}$ et la dose mortelle à $30 \mathrm{mg} \cdot \mathrm{kg}^{-1}$, soit une concentration plasmatique de 4 à $6 \mathrm{mg} \cdot \mathrm{L}^{-1}(14-16)$. La conversion en $\mu$ mol. $\mathrm{L}^{-1}$ est effectuée par la formule : X (mg. $\left.\mathrm{L}^{-1}\right) \times 3,13=\mathrm{Y}\left(\mu \mathrm{mol} . \mathrm{L}^{-1}\right)$ Ces rappels pharmacocinétiques éclairent la symptomatologie et l'approche analytique des intoxications aiguës.

\section{Aspects cliniques}

L'intoxication aiguë est caractérisée par sa gravité, sa soudaineté et la précocité d'apparition des troubles cardiovasculaires ainsi que par son évolution imprévisible : alors que le tableau clinique est rassurant, elle peut évoluer brutalement vers un arrêt cardio-circulatoire.

Les premiers signes cliniques apparaissent 1 à 3 heures après l'ingestion de la molécule et associent troubles cardio-vasculaires, neuro-sensoriels, digestifs, respiratoires et hydro-électrolytiques. Lors d'intoxications massives, ce délai peut être seulement de quelques minutes avec l'apparition d'emblée d'un collapsus voire d'un arrêt cardiaque (14 - 17).

\section{Signes digestifs et neurosensoriels}

Les vomissements sont fréquents. Ils peuvent diminuer de façon importante la quantité de médicament réellement absorbée mais ils exposent au risque d'inhalation chez les sujets comateux. Fgalement précoces, les signes neurologiques sont un précieux élément d'orientation diagnostique. L'atteinte de la $2^{\text {enc }}$ paire crânienne est responsable d'un flou visuel, d'une diplopie, parfois d'une amaurose. L'atteinte chochléo-vestibulaire $\left(8^{\text {imc }}\right.$ paire) entraîne des vertiges, des bourdonnements d'oreille et une hypoacousie. Sont également observés des céphalées, une agitation anxieuse, une obnubilation ou des convulsions (14-17).

\section{Signes cardiovasculaires}

Les signes cardio-vasculaires, liés à l'action quinidinelike de la chloroquine, sont responsables de la gravité de l'intoxication.

Possédant une analogie structurale avec la quinine, la quinidine et les anesthésiques locaux, la chloroquine fait partie de la classe Ia des agents anti-arythmiques de la classification de Vaughan-Williams. Elle possède ainsi une action stabilisatrice de membrane, due à un blocage des canaux sodiques, potassiques et calciques du myocyte. Par dépression du courant sodique rapide, la vitesse de dépolarisation décroît, ce qui entraîne des troubles de la conduction à tous les niveaux. Cette propriété se traduit par des anomalies de l'électrocardiogramme: troubles de la repolarisation (aplatissement de l'onde T, apparition de l'onde U, allongement du QT), troubles de la conduction intraventiculaire (allongement du QRS) et troubles de la conduction auriculoventriculaire (allongement du $P R$, bloc auriculo-ventriculaire) (figure 2). Ces anomalies ont une importante valeur d'alarme.

A forte concentration, la chloroquine est également un agent inotrope négatif et possède une action vasodilatatrice artérielle. L'ensemble de ces mécanismes est à l'origine des perturbations hémodynamiques : bradycardie relative allant jusqu' au collapsus ou à l'arrêt cardio-circulatoire (14-18).

\section{Autres signes}

L'intoxication est parfois responsable d'une polypnée au cours d'intoxications peu graves. Une hypoxémie sans hypercapnie, compatible avec une majoration du shunt intra-pulmonaire par effet vasodilatateur de la chloroquine a également été rapportée (15-17).

Au niveau biologique, l'hypokaliémie, toujours présente dans les intoxications sévères, est parfois très profonde (jusqu'à $0,8 \mathrm{mmol} . \mathrm{L}^{-1}$ ) et peut entraîner une tétraparésie. Elle résulte d'un transfert intracellulaire du potassium par action sur la pompe $\mathrm{Na}^{+} / \mathrm{K}^{+} /$ATPase et n'est donc pas liée à une déplétion potassique. Sa découverte chez un patient intoxiqué doit faire évoquer le diagnostic d'intoxication aiguë à la chloroquine (14-17).

Une hyperlactatémie peut apparaître due à une anomalie de l'utilisation tissulaire de l'oxygène. Enfin, une élévation de la créatinine est également observée et l'installation d'une insuffisance rénale oligo-anurique est de mauvais pronostic (14-17). 
(a)
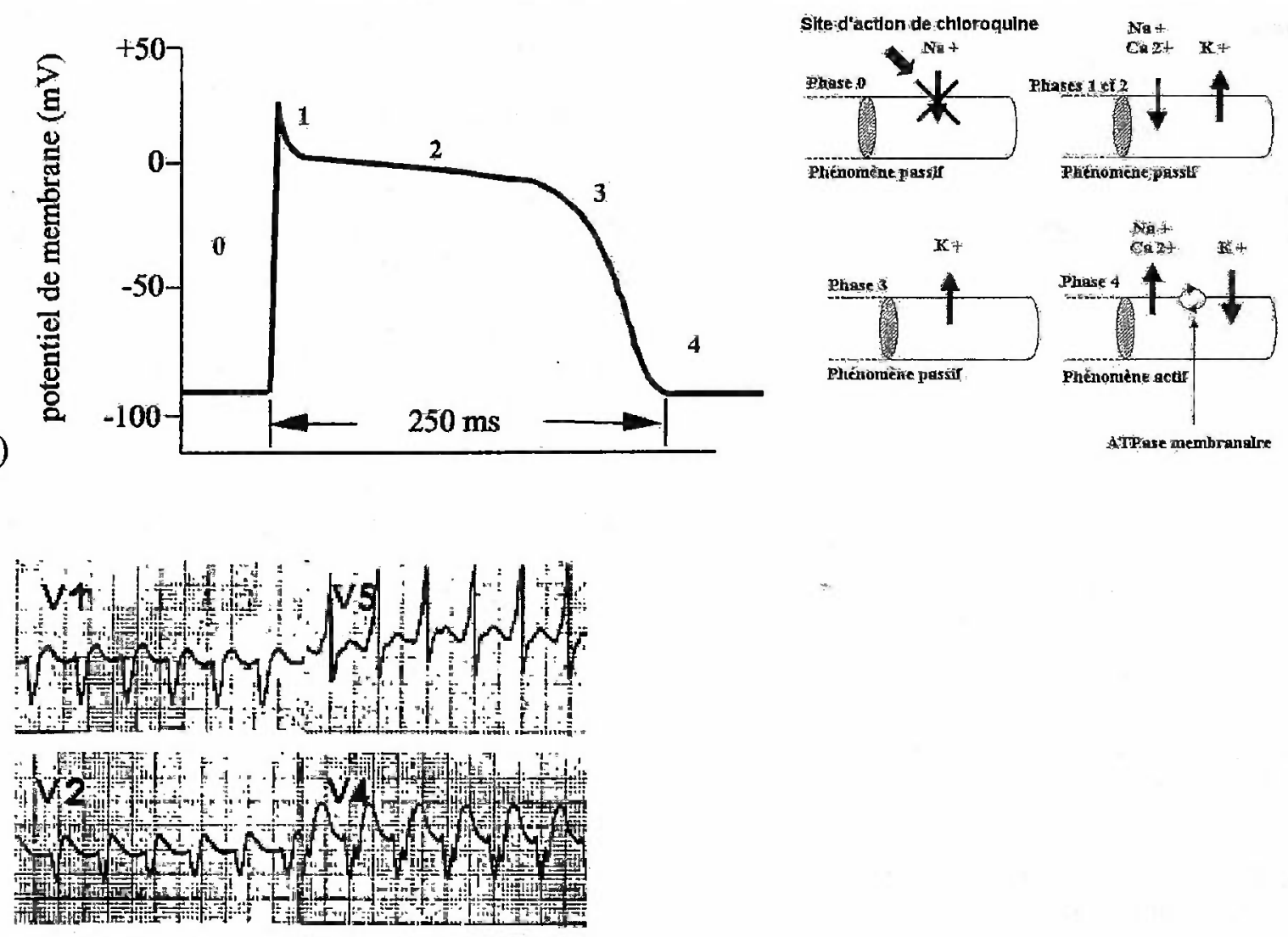

(b)

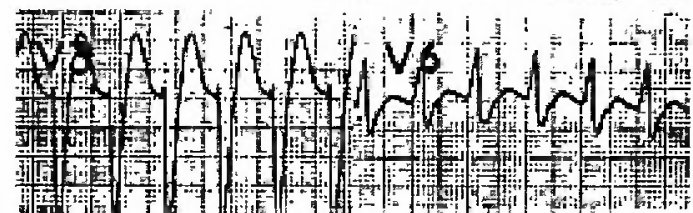

Figure 2 : Mécanisme d'action pharmacologique de la chloroquine au niveau cardiaque (a) et répercutions sur l'E.C.G. (b). Au repos, une différence de potentiel de l'ordre de 80 à $90 \mathrm{mV}$ est observé au niveau des fibres myocardiques : c'est le potentiel de repos. La contraction myocardique est précédée d'une inversion de cette différence de potentiel : le potentiel d'action. Ces phénomènes électriques sont liés à des modifications des courants transmembranaires liés au passage des ions $\mathrm{Na}^{+}, \mathrm{K}^{+}$et $\mathrm{Ca}^{2+}$. La dépolarisation rapide initiale (phase 0) est lié à l'entrée de $\mathrm{Na}^{+}$. Puis le début de la repolarisation (phasel) correspond à l'augmentation de la sortie de $K^{+}$. Cette phase est suivie d'un plateau (phase 2) résultant de l'entrée de Ca ${ }^{2+}$ combinée à la sortie de $K^{+}$. La reprise de polarisation (phase 3) correspond à nouveau à la prévalence des mouvements de $K^{+}$jusqu'à l'atteinte du potentiel de repos (phase 4). Anti-arythmique de classe Ia, la chloroquine possède une action stabilisatrice de membrane provenant principalement du blocage des cannaux sodiques de la cellule cardiaque (inhibition de l'entrée de sodium et de la sortie de potassium avec transfert intracellulaire de calcium). Il en résulte une augmentation la durée du potentiel d'action et de la période réfractaire effective et un allongement de l'espace QT de l'E.C.G. (37). L'E.C.G. présenté a été observé suite à une intoxication par cinq grammes de chloroquine (18).

\section{Prise en charge thérapeu- tique}

\section{Critères de gravité}

L'attitude thérapeutique est fonction de l'évaluation initiale de la gravité de l'intoxication. Celle-ci repose sur l'appréciation de trois paramètres : la dose supposée ingérée, la baisse de pression artérielle systolique et la durée du segment QRS, auxquels il faut ajouter les troubles du rythme, l'hypokaliémie et la chloroquiné-

mie (14-17).

Si les déterminations de la pression artérielle systolique et de la durée du segment QRS ne posent pas de problèmes spécifiques, celle de la dose supposée ingérée est plus aléatoire : l'examen des emballages vides est peu fiable, le patient peut volontairement fournir de fausses informations, les vomissements précoces peuvent minorer la dose réellement absorbée. Dès lors, il n'est pas étonnant de constater que quelle que soit la dose supposée ingérée, la chloroquinémie varie dans une fourchette de 1 à 10 (15). 
Clemessy et al. ont démontré la corrélation existante entre hypokaliémie et gravité de l'intoxication. Dans une série de 191 intoxications, la kaliémie des sujets décédés était significativement plus faible que celle des sujets survivants $\left(2,4 \mathrm{mmol} . \mathrm{L}^{-1}\right.$ contre $3,1 \mathrm{mmol} . \mathrm{L}^{-1}, \mathrm{p}=$ 0,0003) (19).

Le pronostic est également lié à la chloroquinémie. Des concentrations sanguines sur sang total supérieures ou égales à $2 \mathrm{mg} \cdot \mathrm{L}^{-1}$ sont associées à des complications. Actuellement, grâce aux progrès thérapeutiques, la mortalité est nulle en dessous de $4 \mathrm{mg} \cdot \mathrm{L}^{-1}$, de $2 \%$ entre 4 et $8 \mathrm{mg} . \mathrm{L}^{-1}$ et de $21 \%$ au delà de $8 \mathrm{mg} . \mathrm{L}^{-1}(14-16)$.

A partir des éléments précédents, une classification de la gravité des intoxications en trois stades a été proposée : bénigne, intermédiaire et grave (tableau II). Cette évaluation doit être répétée régulièrement afin de mettre en évidence toute aggravation de l'état du patient et donc d'adapter la thérapeutique $(14-15,17)$.

\section{Éléments du traitement}

Le traitement spécifique repose sur la triade diazépam, adrénaline et ventilation assistée.

Le diazépam possède une action favorable au cours des intoxications expérimentales à la chloroquine (augmentation de la pression artérielle, de la fréquence cardiaque, de la diurèse et diminution de la durée du QRS). Cependant, ce bénéfice thérapeutique n'est pas retrouvé lors d'études cliniques. En 1996, Clemessy et al. ont évalué l'intérêt du diazépam versus placebo dans la prise en charge des intoxications de gravité intermédiaire. Cette étude n'a pas mis en évidence une efficacité du diazépam sur la durée du QRS, du QT ou sur la tension artérielle (20): En outre, l'utilisation de diazépam en ventilation spontanée aux posologies préconisées dans la conférence de consensus $(0,5 \mathrm{mg} / \mathrm{kg}$ puis 1 $\mathrm{mg} / \mathrm{kg} / 24 \mathrm{H} 00$ ) expose à une détresse respiratoire et impose donc un monitorage adapté. L'emploi du diazépam en cas d'intoxication bénigne ou intermédiaire n'est donc pas retenu et son mécanisme d'action n'est pas totalement élucidé (interaction avec la cinétique de la chloroquine, anti-arythmique par action sur les récepteurs myocardiques aux benzodiazépines, effet anticonvulsivant).
L'adrénaline, par ses effets $\alpha$ et $\beta$, constitue le traitement de choix de la baisse de l'inotropisme, de la vasodilation artérielle et de la bradycardie liée à la chloroquine. Elle prévient aussi l'apparition de torsades de pointe (14-15).

La ventilation assistée permet l'utilisation en toute sécurité de fortes posologies de diazépam. Elle se justifie également chaque fois que l'hypoxémie persiste malgré une oxygénation suffisante, en cas d'arrêt cardio-respiratoire et de complications pulmonaires (œdème aigu du poumon, syndrome de détresse respiratoire aiguë). Le contrôle des voies aériennes supérieures est licite s'il existe des troubles de la conscience et des vomissements (14-15).

Le traitement spécifique est complété par :

- un traitement évacuateur avec lavage gastrique et utilisation de charbon activé. Cependant, ce traitement n'est recommandé que dans la première heure suivant l'ingestion et ne doit en aucun cas retarder la mise en route du traitement spécifique. Les techniques d'épuration rénale et extra-rénale sont inefficaces en raison de l'importance de la fixation protéique et érythrocytaire de la chloroquine.

- un traitement à visée cardiovasculaire. Le remplissage vasculaire fait partie du traitement de tout patient hypotendu. En raison du l'effet ionotrope négatif de la chloroquine et du risque d'œdème aigu du poumon, ce remplissage doit être modéré (au maximum $1000 \mathrm{~mL}$ de colloïdes). Le choc électrique externe est indiqué pour restaurer un rythme sinusal en cas de tachycardie ou de fibrillation ventriculaire. Le bicarbonate ou le lactate de sodium molaire $(250 \mathrm{~mL}$ répétés 1 fois si nécessaire) sont efficaces sur les blocs de conduction intraventriculaire.

- une induction anésthésique rapide de type «estomac plein» est nécessaire ; l'étomidate étant l'agent préconisé (action rapide avec peu d'effet hémodynamique).

Enfin, la correction de l'hypokaliémie est discutable. L'hypokaliémie étant transitoire, une recharge massive expose à une hyperkaliémie secondaire, surtout en cas d'insuffisance rénale aiguë anurique $(14-15,17)$.

Tableau II : Classification des intoxications aiguës à la chloroquine. La présence d'un seul critère de la troisième colonne suffit pour identifier une intoxication potentiellement fatale (15).

\begin{tabular}{|l|c|c|c|}
\hline & Intoxication "bénigne" & Intoxication "intermédiaire" & Intoxication "grave" \\
\hline Dose supposée ingérée & $\leq 2 \mathrm{~g}$ & $2 \leq<4 \mathrm{~g}$ & $\geq 4 \mathrm{~g}$ \\
\hline Pression artérielle systolique & $\geq 100 \mathrm{mmHg}$ & $\geq 100 \mathrm{mmHg}$ & $<100 \mathrm{mmHg}$ \\
\hline Durée du Q.R.S. & $\leq 0,10 \mathrm{~s}$ & $\leq 0,10 \mathrm{~s}$ & $>0,10 \mathrm{~s}$ \\
\hline Troubles du rythme & - & + & + \\
\hline
\end{tabular}




\section{Modalités d'application de la prise en charge thérapeutique}

La prise en charge thérapeutique a fait l'objet d'une conférence de consensus en 1987 (21). Les recommandations qui y sont proposées ont depuis été modulées. Le protocole de traitement spécifique qui y est mentionné nè doit être appliqué dans son intégralité qu'en cas d'intoxications graves et non systématiquement. En cas d'intoxication bénigne ou intermédiaire, l'hospitalisation en unité de soins intensifs s'impose avec surveillance scopique et réévaluation à intervalles réguliers des critères de gravité de l'intoxication. Toute aggravation doit faire envisager le traitement d'une forme sévère (tableau III). En cas d'intoxication vue après la $6^{\text {emc }}$ heure ces indications doivent être adaptées tout en gardant à l'esprit qu'il est plus dangereux de sous-estimer une intoxication grave que de traiter par excès une intoxication bénigne (15).

Pour être pleinement efficace, le traitement des formes sévères doit être instauré le plus rapidement lors de la phase préhospitalière. Le cas reporté par Messant et al. (11) en 2004 illustre ce propos. Une femme de 25 ans est retrouvée inconsciente dans une chambre d'hôtel et la présence à ses côtés de boîtes vides de chloroquine permet d'orienter l'origine de l'intoxication et d'évaluer la dose ingérée à $10 \mathrm{~g}$. Arrivés rapidement sur les lieux, les secours pratiquent une ventilation artificielle accompagnée de l'administration d'adrénaline et de diazépam. A son admission à l'hôpital, ce traitement est poursuivi et complété d'un traitement épurateur (lavage gastrique et charbon actif). Malheureusement, la patiente présente l'ensemble des critères accompagnant une intoxication fatale : une dose supposée ingérée supérieure à $5 \mathrm{~g}$, une pression artérielle systolique de $80 \mathrm{~mm} \mathrm{Hg}$, une durée du segment QRS de $0,14 \mathrm{~s}$, une chloroquinémie de $12,46 \mathrm{mg} \cdot \mathrm{L}^{-1}$ ainsi qu'une hypo- kaliémie à 1,7 mmol.L $\mathrm{L}^{-1}$. Cependant, une normalisation de sa fonction cardiaque est observée au bout de $24 \mathrm{H} 00$ puis de l'ensemble des paramètres cliniques et biologiques en $48 \mathrm{H} 00$. Selon les auteurs, la rapidité de la prise en charge pré-hospitalière permet d'expliquer l'issue favorable de cette tentative d'autolyse.

\section{Aspects analytiques}

En cas de suspicion d'intoxication aiguë à la chloroquine, le laboratoire possède plusieurs rôles :

- affirmer le diagnostic et évaluer la gravité de l'intoxication par la mise en évidence et le dosage de la chloroquine dans le sang,

- et réaliser des examens complémentaires pour estimer les répercussions de l'intoxication sur l'équilibre hydro-électrolytique (kaliémie) et acido-basique (gazométrie artérielle, dosage des lactates) et sur la fonction rénale (urémie et créatininémie).

Ces éléments ont été rappelés en 2003 par le groupe de travail pluridisciplinaire S.F.B.C. - S.F.T.A. - S.T.C. "Toxicologie et biologie clinique" (22). Seules les données relatives à la mise en évidence et au dosage de la chloroquine seront développées.

\section{Méthodologies décrites dans la littérature}

Le dosage de chloroquine s'effectue sur sang veineux périphérique recueilli sur anticoagulant, l'analyse étant réalisée préférentiellement sur sang total en raison du tropisme de la chloroquine pour les hématies. Quelque soit la technique utilisée, une hydrolyse alcaline du sang total à chaud est nécessaire afin de libérer la molécule de ses liaisons protéiques.

\section{Technique spectrophotométrique}

Après hydrolyse, la chloroquine est extraite de l'échantillon sur colonne Extrelut ${ }^{\mathbb{1}}$ ou en ampoule à décanter

Tableau III : Indications du traitement spécifique des intoxications aiguës à la chloroquine prise en charge avant la $\sigma^{\text {eme }}$ heure (15).

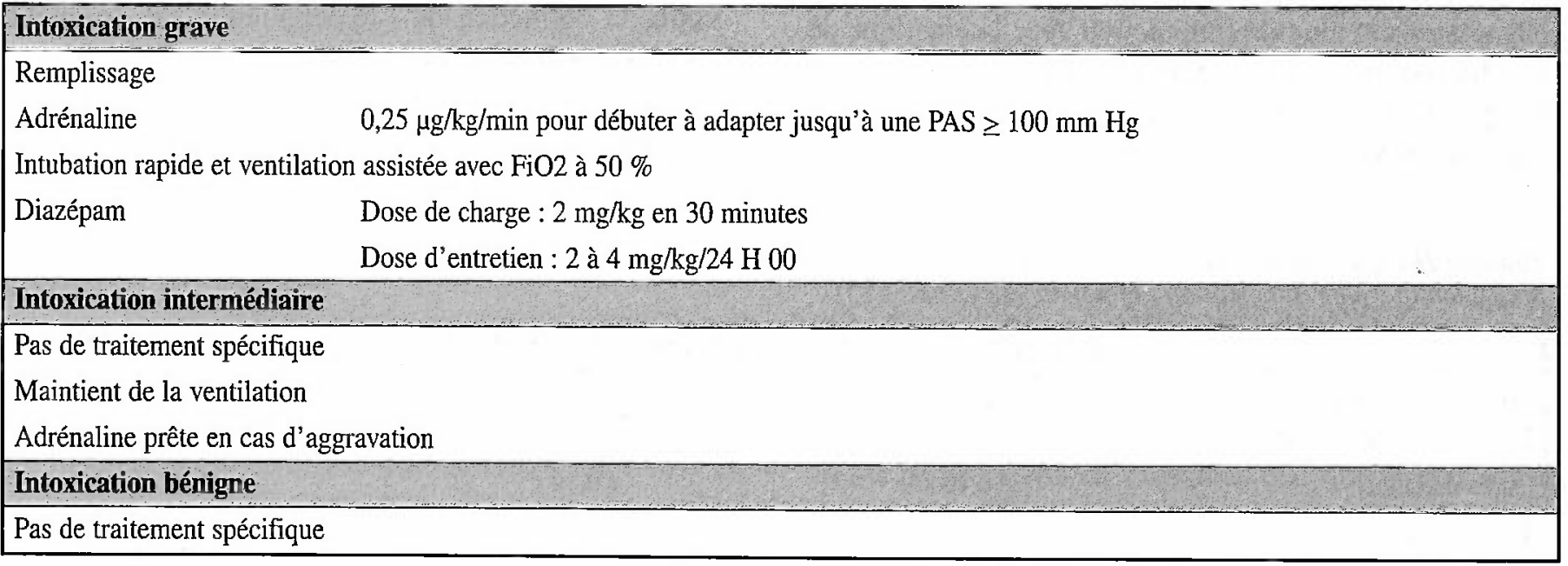


par de l'éther. Des lavages successifs (un lavage par de la soude $0,01 \mathrm{~N}$ puis deux lavages à l'eau distillée) précèdent une contre-extraction par de l'acide sulfurique $(0,5 \mathrm{~N})$. Un spectre UV est ensuite enregistré entre 200 et $400 \mathrm{~nm}$ (figure 3). La réalisation en parallèle d'un spectre témoin, obtenu par surcharge d'un sang avec une solution titrée de chloroquine, permet l'identification et le dosage de la molécule dans l'échantillon. Pour affirmer la présence de chloroquine, le spectre obtenu doit être comparé au spectre caractéristique de la molécule présentant deux pics à 330 et $343 \mathrm{~nm}$ et une vallée à $336 \mathrm{~nm}$. La détermination de la chloroquinémie s'effectue par application de la loi de BEER-LAMBERT à $343 \mathrm{~nm}$.

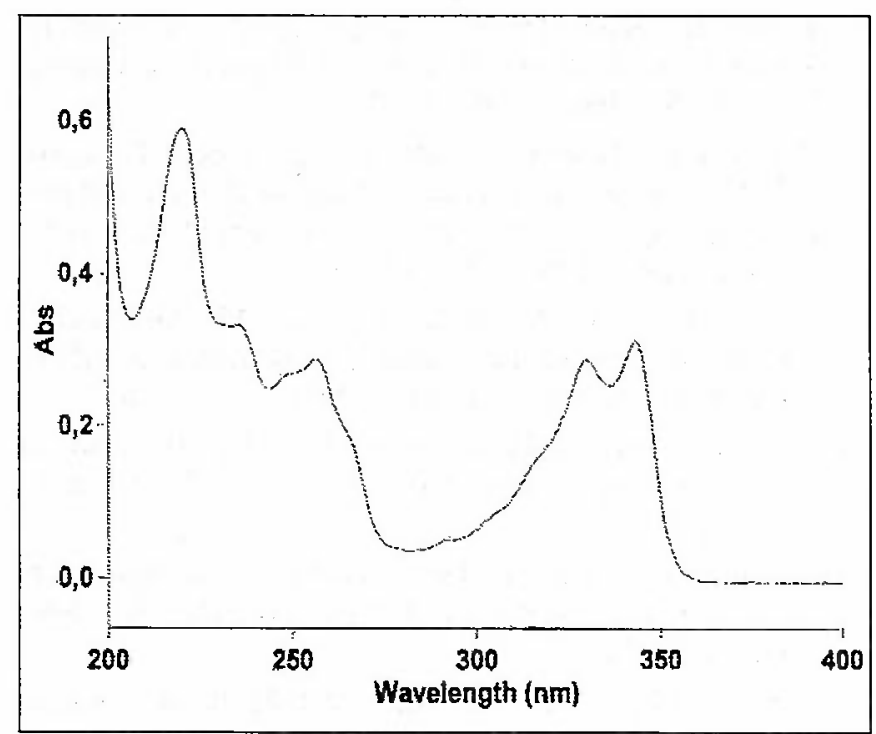

Figure 3 : Spectre d'absorption UV caractéristique de la chloroquine $\left(\mathrm{H}_{2} \mathrm{SO}_{4} ; 0,5 \mathrm{~N}\right)$ avec deux pics d'absorption à 330 et $343 \mathrm{~nm}$ et une vallée à $336 \mathrm{~mm}$.

Cette technique, nécessitant un spectrophotomètre, est simple et rapide à mettre en œuvre. Adaptée à l'urgence (résultat disponible en une heure), elle présente comme inconvénient majeur l'absence d'étalon interne. Dès lors, le rendement d'extraction étant difficile à évaluer, cette méthode de dosage ne permet qu'un dosage semi-quantitatif.

\section{Techniques chromatographiques}

De nombreuses méthodes chromatographiques sont décrites dans la littérature pour le dosage simultané de la chloroquine et de ses principaux métabolites (monodéséthyl- et bidéséthyl-chloroquine). Il existe des variantes permettant également de doser le diazépam afin d'en moduler la posologie (23). Elles font appel à la chromatographie en phase gazeuse avec un détecteur à ionisation de flamme ou couplée à un spectromètre de masse (24) ou à la chromatographie liquide haute per- formance avec un détecteur UV-visible, barrettes de diodes ou fluorimétrique (25-29).

Cependant, la majorité des méthodes ayant été développée dans le cadre d'études thérapeutiques ou pharmacocinétiques, leurs limites de linéarité (de l'ordre du $\left.\mathrm{mg} . \mathrm{L}^{-1}\right)$ sont dépassées lors d'intoxications sévères. En outre, ces techniques, nécessitant un appareillage spécialisé et des techniciens formés, sont difficilement adaptées à l'urgence du fait du délai de rendu de résultats. Elles constituent les méthodes de référence pour les dosages médico-légaux sur des prélèvements sanguins ou tissulaires (cœur, foie, rate, poumons ...).

\section{Autres techniques}

Des méthodes immunologiques, de type ELISA et RIA, permettant de détecter la chloroquine et ses métabolites dans les fluides biologiques (sang et urines) ont été mises au point dans les années 90 (30-32). Sensibles, spécifiques et rapides, elles semblaient prometteuses pour effectuer le diagnostic d'une intoxication aiguë à la chloroquine, mais n'ont pas été commercialisées.

D'autres techniques comme l'électrophorèse capillaire (33) ou, plus anecdoctiquement, la résonance magnétique nucléaire, ont été décrites (34-35). Difficilement adaptables à l'urgence, elles restent réservées à des laboratoires spécialisés.

\section{Pièges interprétatifs}

Si l'interprétation des résultats obtenus en toxicologie clinique ne pose pas de problèmes particuliers, il n'en est pas de même en cas d'expertise médico-légale. Le cas rapporté par Keller et al. en 1998 éclaire parfaitement cet élément. Afin d'établir avec certitude la cause du décès par autolyse d'un homme de 32 ans, des analyses toxicologiques ont été effectuées sur des échantillons pré-mortem (sang veineux, urines et liquide gastrique prélevés à l'admission) et post-mortem (sang périphérique et intracardiaque) à l'aide d'une méthode chromatographique en phase gazeuse couplée à un spectromètre de masse. Les résultats obtenus sont regroupés dans le tableau IV. Si les concentrations veineuses pré-mortem attestent de l'origine de l'intoxication, la différence observée entre la concentration du sang veineux post-mortem $\left(9,2 \mathrm{mg} . \mathrm{L}^{-1}\right)$ et celle du sang cardiaque $\left(26,4 \mathrm{mg} \cdot \mathrm{L}^{-1}\right)$ mérite d'être soulignée. Elle s'explique par une lyse des cellules myocardiques, entraînant une libération de la chloroquine. Le même phénomène est constaté avec les autres toxiques à tropisme cardiaque comme la digoxine, l'imipramine et de certains antidépresseurs (36). Cet évènement peut fausser l'interprétation d'une expertise médico-légale en suggérant que le décès est lié à une intoxication aiguë à la chloroquine alors que le patient suivait tout simplement une chimioprophylaxie antipaludéenne. 
Tableau IV : Concentrations en chloroquine dans différents fluides biologiques observées par Keller et al. lors d'une intoxication fatale à la chloroquine. Les différences de concentration observées dans le sang veineux post-mortem et le sang intracardiaque sont liées à la lyse post-mortem des cellules cardiaques (9).

\begin{tabular}{|l|c|}
\hline Nature du prélèvement et moment du prélèvement & Concentration en chloroquine (mg. L-1) \\
\hline Sang veineux périphérique (admission) & 6,5 \\
\hline Urine (admission) & 683,0 \\
\hline Liquide gastrique (admission) & 310,5 \\
\hline Sang veineux périphérique (post-mortem) & 9,2 \\
\hline Sang intracardiaque (post-mortem) & 26,4 \\
\hline
\end{tabular}

\section{Conclusion}

Molécule à index thérapeutique étroit, la chloroquine est à l'origine d'intoxications aiguës graves. Leur sévérité est essentiellement liée aux troubles cardiovasculaires provoqués par son action quinidine-like. Différents paramètres cliniques (pression artérielle systolique, durée du Q.R.S., troubles du rythme), biologiques (hypokaliémie) et toxicologiques (chloroquinémie) permettent d'évaluer la gravité de l'intoxication et d'orienter la thérapeutique. La prise en charge de cette intoxication fait appel principalement au diazépam, à l'adrénaline et à la ventilation assistée. Cependant, le protocole de traitement spécifique ne doit être appliqué dans son intégralité qu'en cas d'intoxications graves et non systématiquement. Pour être pleinement efficace, il doit être instauré le plus rapidement possible lors de la phase préhospitalière. La chloroquinémie, critère de gravité de l'intoxication, est déterminée préférentiellement sur sang total à l'aide de techniques spectroscopiques (UV) ou chromatographiques (C.L.H.P ou C.P.G.). Si ces dernières constituent les méthodes de référence pour les analyses médico-légales, elles sont difficilement adaptées à l'urgence du fait de leur délai de rendu de résultats.

\section{Références}

1. Anonyme. Monographies Nivaquine ${ }^{\circledR}$. Edition Vidal $2002: 1277-80$.

2. Anonyme. Monographie Savarine ${ }^{\circledR}$. Edition Vidal 2002 : $1630-1$.

3. Lebrun-Vignes B. Les antimalariques : pharmacologie, pharmacocinétique et toxicité chez l'adulte. Med. Mal. Infect. 1999; 29: 229-48.

4. Chitsike I. Acute poisoning in a paediatric intensive care unit in Harare. Cent. Afr. J. Med. 1994 ; 40: 315-9.

5. Ndiaye N., Petrognani R., Diatta B., Seck M. Theobald X., Adnet $\mathrm{P}$. Intoxication à la chloroquine avec détresse respiratoire d'évolution fatale. Ann. Fr. Anesth. Réan. 1999 ; $18: 683-5$.

6. Semb S.O., Jacobsen D. Chloroquine poisoning. Tidsskr. Nor. Laegeforen. 1996; $116: 478-80$.
7. Altrock G, Lange A, Munster P. Acute chloroquine poisoning. Dtsch. Med. Wochenschr. 1997 ; 122 : 225-8.

8. Jahn J., Djonlagic H. Acute chloroquine poisoning. Dtsch. Med. Wocehnschr. 1997 ; 122 : 1232-3.

9. Keller T., Schneider A., Lamprecht R., Aderjan R., Tutsch-Bauer E., Kisser W. Fatal chloroquine intoxication. Forensic. Sci. Inter. $1998 ; 96: 21-8$.

10. Personne M., Rombo L., Sandler H., Wegener T. Serious effect of chloroquine overdose. Prescribe the smallest possible dosage-packages and inform about the risks. Lakartidningen. 1999 ; 96 : 5086-7.

11. Messant I., Jérémie N., Lenfant F., Freysz M. Massive chloroquine intoxication: importance of early treatment and prehospital treatment. Resuscitation. 2004 ; 60 ; 343-6.

12. Riou B., Barriot P., Rimailho A., Baud F.J. Treatment of severe chloroquine poisoning. N. Eng. J. Med. 1988 ; $318: 1-6$.

13. Musshoff F., Madea B. Demonstration of a chloroquine fatality after 10 -month earth-grave. Forensic. Sci. Inter. $2002 ; 125: 201-4$.

14. Carpentier J.P., Rousseau J.M., Imbert M. Intoxication par la chloroquine. In : J.M. Saissy. Urgences et réanimation en milieu militaire. Reuil Malmaison : Arnette Groupe Liaisons SA, 2000 ; 475-86.

15. Clemessy J.L., Lapostolle F., Borron S.W., Baud F.J. Intoxication aiguë à la chloroquine. Presse Med. 1996 ; $25: 1435-39$.

16. Bismuth C. Toxicologie clinique 5ème Edition. Paris : Médecine - Sciences Flammarion ; 2000 : 366-9.

17. Riou B., Barriot P. Intoxication aiguë par la chloroquine. Médecine thérapeutique. $1999 ; 5: 37-42$.

18. Queyriaux B., Carlmioz R., Perrier E., Micaelli P., Gressard A., Deroche J., Leduc P.A., Quiniou G., Burlaton J.P. Les effets cardiovasculaires liés à I'utilisation de la chloroquine. Ann. Cardiol. Angéiol. 2001 ; 50 : 285-92.

19. Clemessy J.L., Favier C., Borron S.W., Hantson P.E., Vicaut E., Baud F.J. Hypokalaemia related to acute chloroquine ingestion. Lancet. $1995 ; 346: 877-80$.

20. Clemessy J.L., Favier C., Borron S.W. Therapeutic trial of diazepam versus placebo in acute chloroquine intoxications of moderate gravity. Intensive Care Med. 1996 ; 22 : 1400-5.

21. Conférence de consensus Société de Réanimation de Langue Française. Traitement de l'intoxication aiguë à la chloroquine. Réanim. Soins Intens. Med. Urg. 1988 ; 318 : 1-6. 
22. Groupe de travail plutidisciplinaire SFBC-SFTA-STC «Toxicologie et biologie clinique». Biomarqueurs de toxicité et anomalies métaboliques dans les principales intoxications graves. Symptomatologie clinique et toxique. Le prélèvement conservatoire. Ann. Biol. Clin. 2003 ; 61 : 421-33.

23. Estadieu M., Durand A., Viala A., Rop P.P., Fornaris M., Quicke J. A rapid HPLC procedure for simultaneous determination of chloroquine, monodesethylchloroquine, diazepam and nordiazepam in blood. J. Anal. Toxicol. $1989 ; 13: 89-93$.

24. Bergqvist Y., Eckerborn S. An improved gas chromatographic method for the simultaneous determination of chloroquine and two metabolites using capillary columns. J. Chromatogr. 1984 ; 306 : 147-53.

25. Lambert W.E., Van Bocxlaer J.F., De Leenheer A.P. Potential of high-performance liquid chromatography with photodiode array detection in forensic toxicology. J. Chromatogr. B. Biomed. Sci. Appl. 1997 ; 689 : 45-53.

26. Volin P. Simple and specific reversed-phase liquid chromatographic method with diode array detection for simultaneous determination of serum hydroxychloroquine, chloroquine and some corticosteroids. J. Chromatogr. B. 1995 ; 666 : 347-53.

27. Augustijns P. Determination of chloroquine and desethylchloroquine in biological samples using perfusion chromatography and fluorescence detection. J. Liq. Chrom. Rel. Technol. 1997 : 20 ; 1103-13.

28. Walker O., Ademowo O.G. A rapid cost effective liquid chromatographic method for the determination of chloroquine and desethylchloroquine in biological fluids. Ther. Drug. Monit. 1996 ; 18 : 92-6.

29. Houze P., De Reynies A., Baud F.J., Benatar M.F., Pays M. Simultaneous determination of chloroquine and its three metabolites in human plasma, whole blood and urine by ion-pair high-performance liquid chromatography. J. Chromatogr. $1992 ; 574: 305-12$.
30. Witte A.M., Klever H.J., Brahin B.J. Eggelte T.A., Van der Kaay, Alpers M.P. Field evaluation of the use an ELISA to detect chloroquine and its metabolites in blood, urine and breast-milk. Trans. R. Soc. Trop. Med. Hyg. 1990 ; 84 : 521-5.

31. Escande C., Chevalier P., Verdier F., Bourdon R. Sensitive radioimmunoassay and enzyme-linked immunosorbent assay for the simultaneous determination of chloroquine and its metabolites in biological fluids. J. Pharm. Sci. $1990 ; 79: 23-7$.

32. Schwick P., Eggelte T.A., Hess F., Tueumuna T.T., Payne D., Nothdurft H.D., Von Sonnenburg F., Loscher T. Sensitive ELISA dipstick test for the detection of chloroquine in urine under field conditions. Trop. Med. Int. Health. 1998 ; 3 : 828-32.

33. Muller D., Blaschke G. Enantioselective assay of chloroquine and its main metabolite deethylchloroquine in human plasma by capillary electrophoresis. J. Chromatogr. Sci. $2000 ; 38: 435-40$.

34. Azaroual N., Deveaux M., Hedouin V., Luneau E., Imbenotte M., Vermeersch G., Gosset D., Lhermitte M. Intoxication par la chloroquine: analyse des fluides biologiques par RMN $1 \mathrm{H}$ et HPLC-DAD. Toxicorama. 1997 ; $9: 371$.

35. Maschke S., Azaroual N., Wieruszeski J.M., Lippens G., Imbenotte M., Mathieu D., Vermeersch G., Lhermitte M. Diagnosis of a acute chloroquine poisoning using $1 \mathrm{H}$ NMR spectroscopy : characterisation of drug metabolites in urine. NMR Biomed. $1997 ; 10: 277-284$.

36. Tracqui A., Ludes B. De l'autopsie au prélèvement. In : P. Kintz. Toxicologie et pharmacologie médico-légales. Paris : Elsevier, 1998 ; 15-26.

37. Motté G., Sebag C, Slama M. Antiarythmiques. Encycl Méd Chir (Elservier, Paris), Cardiologie-Angéiologie, 11 904-A-10, 1996, 8p. 